\title{
The Current State of Research Regarding the Role of Non-Coding RNAs in Cutaneous Squamous Cell Carcinoma
}

This article was published in the following Dove Press journal: OncoTargets and Therapy

\author{
Shuang Chen' \\ Limin Luo ${ }^{2}$ \\ Hongduo Chen (iD) \\ Chundi $\mathrm{He}^{\text {l }}$ \\ 'Department of Dermatology, No.I \\ Hospital of China Medical University, Key \\ Laboratory of Immunodermatology, \\ Shenyang, Liaoning I I000I, People's \\ Republic of China; ${ }^{2}$ Department of \\ Dermatology, The First Affiliated \\ Hospital, Zhejiang University School of \\ Medicine, Hangzhou 310003, People's \\ Republic of China
}

\begin{abstract}
Skin cancers, including those of both both melanoma and non-melanoma subtypes, remain among the most common forms of human cancer. Non-melanoma skin cancers are typically further differentiated into the basal cell carcinoma and cutaneous squamous cell carcinoma (cSCC) categories. Current approaches to diagnosing and treating $\mathrm{cSCC}$ remain unsatisfactory, and the prognosis for patients with this disease is relatively poor. Recent advances in high-throughput sequencing have led to an increasingly robust understanding of the diversity of non-coding RNAs (ncRNAs) expressed in both physiological and pathological contexts. These ncRNAs include microRNAs, long ncRNAs, and circular RNAs, all of which have been found to play key functional roles and/or to have value as diagnostic biomarkers or therapeutic targets in a range of different disease contexts. The number of ncRNAs associated with cSCC continues to rise, and as such, there is clear value in comprehensively reviewing the functional roles of these molecules in this form of cancer in order to highlight future avenues for research and clinical development.
\end{abstract}

Keywords: cSCC, no-coding RNA, miRNA, lncRNA, circRNA

\section{Introduction}

Skin cancer is a highly prevalent disease in humans and is typically classified into melanoma and non-melanoma categories, with the latter category including the basal cell carcinoma (BCC) and cutaneous squamous cell carcinoma (cSCC) skin cancer subtypes. ${ }^{1}$ cSCC remains the second most common skin cancer subtype, accounting for $20 \%$ of all skin cancer deaths globally, with 200,000 newly diagnosed cases annually in the United States alone. ${ }^{2-4}$ Recent advances in surgical, radiotherapeutic, and chemotherapeutic interventions have led to some improvements in $\mathrm{cSCC}$ patient prognosis, but at present, the prognosis for patients with this disease remains relatively poor. As such, it is vital that further studies continue to explore the mechanistic basis for $\mathrm{cSCC}$ in order to identify novel diagnostic, prognostic, and therapeutic targets that can guide patient treatment and improve survival outcomes.

Advances in next-generation sequencing (NGS) technologies in recent decades have led to the identification of countless distinct non-coding RNA transcripts within cells, including microRNAs (miRNAs), long non-coding RNAs (lncRNAs), and circular RNAs (circRNAs). The best understood of these molecules are miRNAs, which are short RNAs ( $22 \mathrm{nt}$ ) capable of directly binding to complementary segments in the 3 '-untranslated region (UTR) of target mRNA molecules in order to regulate gene
Department of Dermatology, No. Hospital of China Medical University, Key Laboratory of Immunodermatology,

Shenyang, Liaoning II000I, People's

Republic of China

Email cdhe@cmu.edu.cn
OncoTargets and Therapy 2020:13 |3|5|-13158

$|3| 5 \mid$

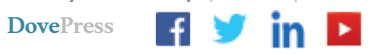

http://doi.org/| $0.2147 /$ TTT.S27|346 
expression. $^{5-7}$ Studies of miRNAs thus typically focus on identifying the target genes governed by these non-coding RNA molecules. In contrast, IncRNAs are significantly longer (>200 nt) and were previously regarded as nonfunctional or "junk" RNA molecules. ${ }^{8,9}$ However, more recent studies have found that IncRNAs are important in both physiological and pathological contexts, wherein they can play diverse roles including modulating mRNA splicing, regulating chromatin remodeling, sequestering miRNAs, or binding to and altering the subcellular localization of specific proteins. ${ }^{8-11}$ cirRNAs were first detected in studies of viral particles, ${ }^{12}$ with further research revealing them to be produced by the back-splicing of mono-exonic, multi-exonic, or intronic gene regions. Because they lack a poly-A tail, these RNA molecules are better able to resist exonucleasemediated degradation than are their linear counterparts. ${ }^{13}$ Although not as well understood as miRNAs and lncRNAs, circRNAs are also thought to play important roles in diseases, wherein they can act as competing endogenous RNA (ceRNA) sponges capable of sequestering specific RNAs, ${ }^{14,15}$ interact with RNA binding proteins, ${ }^{16,17}$ and modulate coding peptides. ${ }^{18,19}$ Countless studies have examined the roles of particular non-coding RNA molecules in specific cancers, revealing them to have value as diagnostic or prognostic biomarkers and to represent potentially viable therapeutic targets. ${ }^{20-22}$ In the present review, we specifically discuss recent studies regarding the role of non-coding RNA molecules in cSCC, we discuss the latest research advance of non-coding RNAs involved in cSCC, with the goal of highlighting key clinically relevant targets that may help to guide patient diagnosis and treatment. In addition, most of these non-coding RNAs may be of value for the diagnosis and treatment of cSCC.

\section{miRNAs in CSCC}

\section{miR-2I}

The overexpression of miR-21 has been found to be a hallmark of several different tumor types, including cSCC, wherein it has been shown to drive the enhanced invasion and metastasis of these tumor cells引用文献PMID: 32,360,623, $31,371,997$. Well-characterized targets of miR-21 with known relevance to cancer development and progression include phosphatase tensin homolog (PTEN) and programmed cell death 4 (PDCD4), with miR-21 leading to the downregulation of both of these tumor suppressor genes. Indeed, in a study of A431 cells, $\mathrm{Li}$ et al found that silencing miR-21 ultimately led to the enhanced expression of both PDCD4 and PTEN in these cells. ${ }^{23}$ Consistent with these observations, Charbel et al found that miR-21 was able to suppress the activity of the GRHL3PTEN axis, which in turn bolstered PI3K/Akt/mTOR signaling in these cells and thereby drove cSCC development and progression. $^{24}$ These previous findings thus highlight the potential for miR-21 to serve as a viable therapeutic target for the treatment of cSCC.

\section{miR-365}

Several studies have identified an oncogenic role for miR$365 .^{25-27}$ Ding et al found that the UVB irradiation of murine NIH3T3 cells was sufficient to induce the upregulation of this miRNA, suggesting that its expression may be closely linked to UV radiation exposure. They further demonstrated that miR-365 was capable of promoting oncogenesis in vitro and in vivo at least in part via targeting nuclear factor $\mathrm{I} / \mathrm{B}$ (NFIB), which is a tumor suppressor gene that can promote the cell cycle arrest and apoptotic death of malignant cSCC cells. ${ }^{28,29}$ As such, this miR-365/NFIB axis may be amenable to therapeutic intervention in $\mathrm{CSCC}$ patients.

\section{miR-3I}

Unlike the two miRNAs discussed above, miR-31 has been shown to play distinct roles in different subtypes of cancer. In breast cancer, for example, reduced miR-31 expression levels are associated with higher rates of metastasis, whereas studies of colorectal cancer suggest that miR-31 overexpression is linked to enhanced invasion and more advanced TNM stage. ${ }^{30,31}$ Work by Wang et al indicated that miR-31 expression levels were increased in cSCC, wherein this miRNA was able to enhance tumor cell proliferation and migration, highlighting it as a potentially viable therapeutic target in this cancer type. $^{32}$ The variable expression levels and inconsistent molecular functions of miR-31 in different cancers indicate that it is associated with more pronounced tissue specificity and may exhibit promising biomarker capacity.

\section{miR-34a}

Lefort et al were the first to highlight a potential role for miR-34a in cSCC, as they found that the expression of this miRNA rose during the process of keratinocyte differentiation, whereas its expression was reduced in SCC cell lines and primary tumor samples, as well as in abnormally differentiating primary human keratinocytes (HKCs). These researchers further identified SIRT6 as a miR-34a target gene in HKCs, with the knockdown of SIRT6 expression being sufficient to enhance cellular proliferation, yielding a phenotype comparable to that observed 
upon miR-34a upregulation. ${ }^{33}$ These findings further highlight this miR-34a/SIRT6 axis as another potential driver of cSCC development that may amenable to therapeutic targeting.

\section{miR- $125 b$}

$\mathrm{Xu}$ et al first reported the downregulation of miR-125b in cSCC tissue samples, and they further demonstrated that in these cSCC cells this miRNA was capable of suppressing tumor cell proliferation, migration, and invasion. ${ }^{34}$ They identified matrix metallopeptidase 13 (MMP13) as a miR$125 \mathrm{~b}$ target gene, with reduced miR-125b expression in cSCC thus leading to increased MMP13 expression. These results, therefore, indicate that miR-125b functions as a tumor suppressor miRNA in cSCC, and may thus be of value as a diagnostic or prognostic biomarker in patients with this disease.

\section{miR-199a}

A number of different tumor types have been found to exhibit miR-199a dysregulation. ${ }^{35,36}$ Research by Wang et al suggested that the expression of this miRNA was markedly reduced in $\mathrm{cSCC}$ and that it was negatively correlated with CD44 expression. ${ }^{37}$ As CD44 encodes a cell surface glycoprotein that is involved in adhesion, migration, and metastasis, this work thus suggested that miR-199a directly regulates CD44 expression and thus represents a viable means of targeting CD44 in the context of cSCC.

\section{IncRNAs in cSCC}

\section{Hotair}

HOTAIR (HOX transcript antisense intergenic RNA) is a lncRNA that is encoded in the 12q13.13 region of the human genome and that is closely linked to the onset of cervical and breast cancers, wherein it can promote tumor cell migration and proliferation. ${ }^{38,39}$ Work by Sand et al has further demonstrated that HOTAIR upregulation is evident in cSCC, with these results being confirmed both via IncRNA microarray and qRT-PCR. ${ }^{40}$ More recently, $\mathrm{Yu}$ et al confirmed that $\mathrm{cSCC}$ cell lines exhibit HOTAIR upregulation, which in turn enhances their migratory and proliferative activity and bolsters their ability to undergo the epithelial-mesenchymal transition (EMT). ${ }^{41}$ HOTAIR is believed to function as a ceRNA capable of sequestering miRNAs including miR-326, which leads to the increased expression of the miR-326 target gene PRAF2 and results in enhanced cell migration and proliferation. This HOTAIR/miR-326/PRAF2 axis therefore offers key insights into the mechanistic basis for cSCC.

\section{TINCR}

The use of photodynamic therapy (PDT) as a minimallyinvasive treatment for premalignant and malignant skin lesions has been increasingly explored in recent years, as this technique does not induce scar formation. SCCs have successfully been treated via ALA (5-aminolevulinic acid)-PDT, with such treatment inducing autophagy and the apoptotic death of malignant cells. The $3.7 \mathrm{~kb}$ lncRNA known as terminal differentiation-induced ncRNA (TINCR) has been shown to mediate the posttranscriptional regulation of human epidermal cellular differentiation. ${ }^{42}$ Research by Zhou et al suggests that ALA-PDT can promote enhanced expression of TINCR via an ERK1/2-SP3 pathway, whereupon it was capable of inducing apoptosis and autophagy in A431 cells. ${ }^{43}$ This thus suggests that TINCR represents a viable lncRNA target for the therapeutic treatment of cSCC.

\section{LINCOI048}

Chen et al identified the lncRNA LINC01048 as being significantly upregulated in recurrent $\mathrm{CSCC}$ tissue samples relative to non-recurrent cSCC or paracancerous normal tissues. ${ }^{44}$ They further found that LINC01048 expression was negatively correlated with overall survival and cure rates in $\mathrm{CSCC}$ patients. At a mechanistic level, they found that knocking down LINC01048 was sufficient to impair cSCC cell growth in vitro and in vivo. Specifically, they determined that LINC01048 upregulation was driven by USF1, and once upregulated, LINC01048 was able to bind to TAF15 in order to drive enhanced YAP1 expression. This study thus highlights the potential relevance of LINC01408 in the context of cSCC, making it yet another potentially valuable diagnostic and or therapeutic target in individuals with this disease.

\section{LINC00520}

Mei et al established LINC00520 as a tumor suppressor lncRNA that is downregulated in cSCC, and that impairs cSCC cell growth and metastasis in vitro and in vivo. ${ }^{45}$ They further found that LINC00520 functioned at least in part by targeting and inhibiting EGFR (epidermal growth factor receptor) expression and thereby disrupting the downstream PI3K/Akt signaling that is essential to robust tumor cell growth. Together, these results thus highlight LINC00520 as another promising biomarker of cSCC. 


\section{PICSAR}

The overexpression of LINC00162 in cSCC cell lines was originally reported by Piipponen et al, who detected this dysregulation through whole transcriptome analysis. They then designated this IncRNA as p38 inhibited cSCCassociated lncRNA (PICSAR). ${ }^{46}$ When these researchers knocked down PICSAR, they found that this significantly impaired cSCC cell growth and migration in vivo and in vitro. They further determined that this IncRNA functions at least in part via inhibiting the MAPK phosphatase DUSP6, thereby leading to enhanced ERK1/2 pathway activation and more robust tumor cell growth. PICSAR thus represents another ncRNA with the potential to serve as a diagnostic biomarker or therapeutic target in $\mathrm{CSCC}^{47}$

\section{AKI4484 I}

Ponzio et al first detected the upregulation of the lncRNA AK144841 in cSCC cells. ${ }^{48}$ While AK144841 expression was largely absent in normal murine skin, it was markedly upregulated in murine cSCC cells. They further found that AK144841 was able to inhibit the expression of several different tumor suppressor genes, including members of the late cornified envelope-1 (Lce1) family, as well as Cgref1, Brsk1, Basp1, Dusp5, and Btg2, with AK144841 expression being negatively correlated with that of these genes. These researchers additionally determined that a human isoform of AK144841 was similarly upregulated in cSCC tissue samples, suggesting that detecting hAK144841 expression in precancerous lesions may allow for the more efficient detection of potentially malignant tumors, thereby guiding clinical treatment strategies.

\section{circRNAs in cSCC} hsa_circ_0070934

At present, relatively limited research has been conducted exploring the functional relevance of circRNAs in cSCC. Work by Sand et al conducted in 2015 sought to characterize circRNA expression profiles in cSCC and normal

Table I Overview of the ncRNAs Have Been Reported in Cutaneous Squamous Cell Carcinoma

\begin{tabular}{|c|c|c|c|c|}
\hline ncRNA & Related Targets & Function & Regulation & Refs \\
\hline miR-2I & PTEN, PDCD4 & Oncogenic & Up-regulation & {$[23,24]$} \\
\hline $\operatorname{miR}-365$ & NIFB & Oncogenic & Up-regulation & {$[28,29]$} \\
\hline miR-3I & ITGA5, RDX WAVE3 & Oncogenic & Up-regulation & {$[32]$} \\
\hline miR-34a & SIRT6 & Tumor suppressor & $\begin{array}{l}\text { Down- } \\
\text { regulation }\end{array}$ & [33] \\
\hline miR-I25b & MMPI3 & Tumor suppressor & $\begin{array}{l}\text { Down- } \\
\text { regulation }\end{array}$ & [34] \\
\hline miR-199a & CD44 & Tumor suppressor & $\begin{array}{l}\text { Down- } \\
\text { regulation }\end{array}$ & [37] \\
\hline HOTAIR & miR-326/PRAF2 & Oncogenic & Up-regulation & {$[40,4 I]$} \\
\hline TINCR & ERKI/2-SP3 pathway & promote ALA-PDT-induced apoptosis and autophagy & $\begin{array}{l}\text { Down- } \\
\text { regulation }\end{array}$ & [43] \\
\hline LINC0I048 & TAFI5/YAPI & Oncogenic & Up-regulation & {$[44]$} \\
\hline LINC00520 & EGFR & Tumor suppressor & $\begin{array}{l}\text { Down- } \\
\text { regulation }\end{array}$ & {$[45]$} \\
\hline PICSAR & DUSP6 & Oncogenic & Up-regulation & {$[46]$} \\
\hline AKI4484I & Lcel, Cgref, BrskI, Baspl, Dusp5, Btg2 & Oncogenic & Up-regulation & {$[48]$} \\
\hline hsa_circ_0070934 & miR-I238/miR-I247-5p & Oncogenic & Up-regulation & {$[50]$} \\
\hline hsa_circ_001937 & miR-597-3p/FOSL2 & Oncogenic & Up-regulation & {$[5 ।]$} \\
\hline circPVTI & NA & Oncogenic & Up-regulation & {$[52]$} \\
\hline
\end{tabular}


paracancerous tissue samples, leading to the identification of 143 and 179 circRNAs that were significantly up- and down-regulated in cSCC tissues, respectively (foldchange $\geq 2$ and $\mathrm{p}<0.05) .{ }^{49}$ The authors further employed an Arraystar miRNA target prediction software in order to identify the miRNA targets of these circRNAs. While this study highlighted the differential expression of circRNAs in $\mathrm{cSCC}$, the authors did not conduct further functional studies of the role of these molecules in this disease context.

In an effort to expand on these above results, An et al identified hsa_circ_0070934 from among the circRNAs that were upregulated in cSCC in the above study and analyzed the functional relevance of this circRNA in depth. ${ }^{50}$ They were able to confirm that hsa_circ_0070934 expression was elevated in both cSCC tissues and cells via qRT-PCR. From a functional perspective, they further found that elevated expression of hsa_circ_0070934 led to markedly enhanced cSCC cell proliferation, migration, and invasion. Using dual-luciferase reporter constructs, they were also able to confirm that hsa_circ_0070934 was able to directly interact with miR-1238 and miR1247-5p, although they did not identify the relevant targets of these miRNAs nor did they rule out the possibility that this circRNA plays additional functional roles in $\mathrm{cSCC}$. These results thus highlight the potential functional relevance of circRNAs in cSCC, although more research will be needed to understand the magnitude of their relevance in this disease context.

\section{hsa_circ_001937}

hsa_circ_001937 was identified as a 2850 nucleotide exonic circRNA that is encoded on chromosome 16引用PMID: 30,259,364. Gao et al identified differentially expressed circRNAs in cSCC using the Arraystar Human circRNA chip引用PMID: $33,000,177$, leading to the discovery that

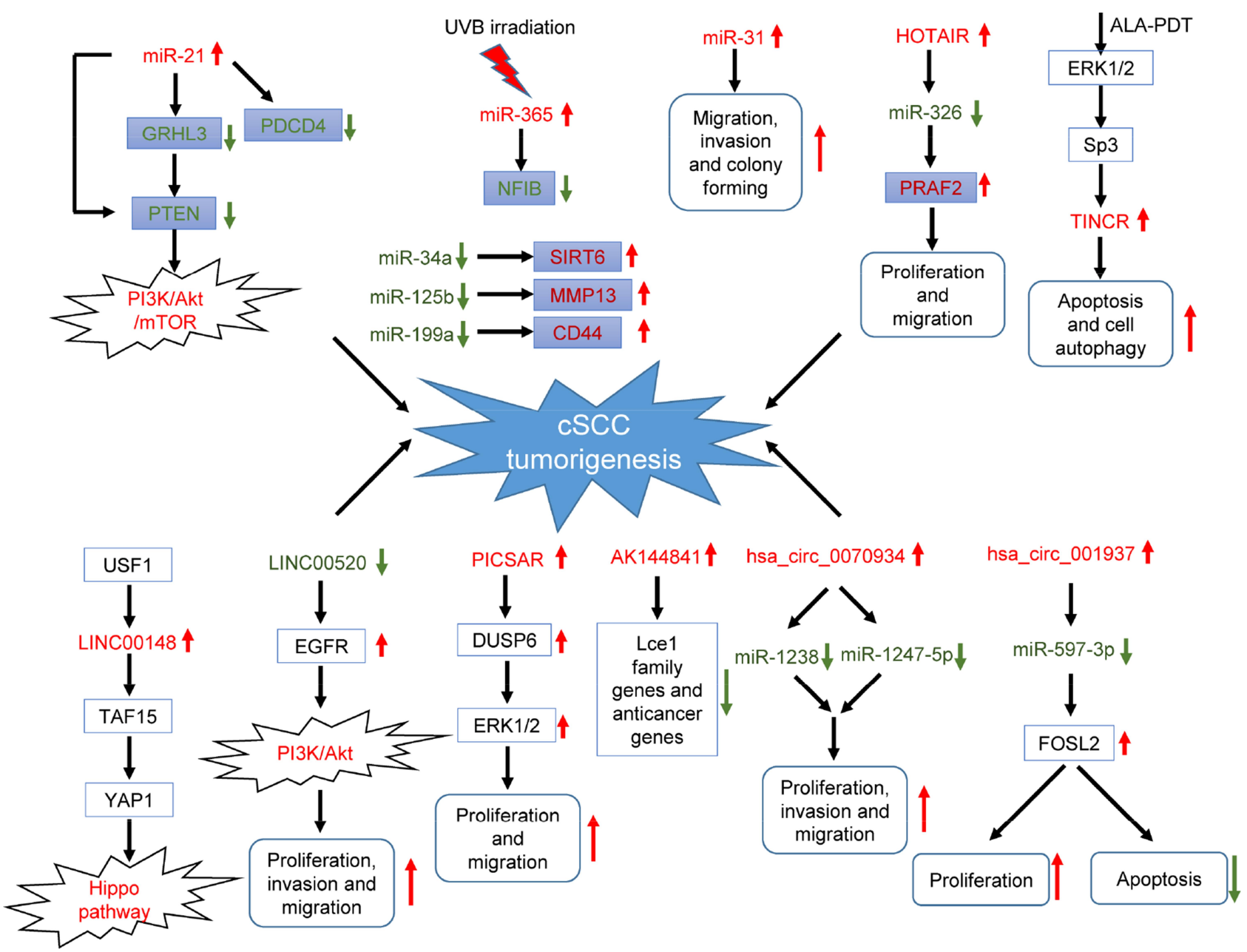

Figure I The biological roles of non-coding RNAs in CSCC. 
hsa_circ_001937 expression was upregulated by more than 14-fold in cSCC tissues. From a mechanistic perspective, the authors determined that hsa_circ_001937 was able to act as ceRNA to sequester miR-597-3p, thereby modulating FOSL2 expression in these cells. As such, the hsa_circ_001937/miR597-3p/FOSL2 axis influences cSCC progression and represents a potential therapeutic target for cSCC patients.

\section{circPVTI}

The 410 nucleotide-long circPVT1 originates from exon 2 of the PVTl gene. In our previous study, we conducted circRNA sequencing using 3 pairs of cSCC tissues in order to profile cSCC-specific circRNAs引用PMID: 32,764,965. A total of 449 dysregulated circRNAs were identified through this analysis, and we found that circPVT1 was upregulated 5-fold in cSCC tissues. We further confirmed the upregulation of this circRNA in relevant tumor cell lines and in 30 pairs of tumor and paracancerous tissues via qRTPCR. Notably, circPVT1 has been shown to be overexpressed and to function as an oncogene in multiple cancers 引用PMID: 30,590,312, 27,986,464, indicating that it may similarly play an oncogenic role in cSCC. In vitro assays revealed that silencing circPVT1 inhibited the migration and invasion of cSCC cells. A ceRNA network was additionally constructed to explore circRNA-miRNA-mRNA interactions, leading to the conclusion that circPVT1 was significantly involved in this regulatory network. These findings indicated that circPVT1 was also able to promote cSCC progression via sequestering target miRNAs and thereby influencing the expression of specific mRNAs. However, further in vitro/in vivo assays are requires to confirm the mechanistic basis for these observations, and these assays represent the focus of our ongoing work.

\section{Concluding Remarks}

In this review, we have provided a comprehensive overview of non-coding RNAs that have been found to be related to the development and/or progression of cSCC (Table 1 and Figure 1). While the mechanisms whereby miRNAs function are fairly well understood, the functionality of lncRNAs and circRNAs in the context of oncogenesis remains to be fully explored. As highlighted in the present article, these noncoding RNAs have the potential to serve as diagnostic and/or therapeutic biomarkers in cSCC, and they additionally offer broad mechanistic insights into transcriptomic functionality in human cells. As NGS technologies continue to advance, additional ncRNAs will likely be identified and better characterized. Further studies focused on the role of circRNAs in
cSCC will be invaluable, as these ncRNAs are poorly studied in this disease context. In addition, some reports suggest that in certain contexts, ncRNAs can be translated into functional proteins or polypeptides, ${ }^{51-56}$ thus highlighting another potential mechanism whereby these molecules can modulate oncogenesis. Further research regarding these mechanisms will prove essential to advancing present understanding of cSCC development and treatment.

\section{Acknowledgments}

This work was supported by a grant from: the Science \& Technology Fund of Liaoning Province (grant no. 201501013); the Distinguished Professor Foundation of Liaoning Province (grant no. Liao (2012)145).

\section{Author Contributions}

All authors made a significant contribution to the work reported, whether that is in the conception, study design, execution, acquisition of data, analysis and interpretation, or in all these areas; took part in drafting, revising or critically reviewing the article; gave final approval of the version to be published; have agreed on the journal to which the article has been submitted; and agree to be accountable for all aspects of the work.

\section{Disclosure}

The authors report no potential conflicts of interest.

\section{References}

1. Bath-Hextall F, Nalubega S, Evans C. The needs and experiences of patients with skin cancer: a qualitative systematic review with metasynthesis. Br J Dermatol. 2017;177(3):666-687. doi:10.1111/ bjd. 15148

2. Scotto J, Kopf AW, Urbach F. Non-melanoma skin cancer among caucasians in four areas of the United States. Cancer. 1974;34 (4):1333-1338. doi:10.1002/1097-0142(197410)34:4<1333::AIDCNCR2820340447>3.0.CO;2-A

3. Rowe DE, Carroll RJ, Day CL. Prognostic factors for local recurrence, metastasis, and survival rates in squamous cell carcinoma of the skin, ear, and lip. J Am Acad Dermatol. 1992;26(6):976-990. doi:10.1016/ 0190-9622(92)70144-5

4. Dziunycz PJ, Lazarova Z, Duncan N, et al. EGFRvIII expression in squamous cell carcinoma of the skin. JAMA Dermatol. 2013;149 (10):1240. doi:10.1001/jamadermatol.2013.5230

5. Forlenza GP, Buckingham BA, Christiansen MP, et al. Performance of omnipod personalized model predictive control algorithm with moderate intensity exercise in adults with type 1 diabetes. Diabetes Technol Ther. 2019;21(5):265-272. doi:10.1089/dia.2019.0017

6. Yu X, Li Z, Liu J. MiRNAs in primary cutaneous lymphomas. Cell Prolif. 2015;48(3):271-277. doi:10.1111/cpr.12179

7. Li Z, Yu X, Shen J, Wu WKK, Chan MTV. MicroRNA expression and its clinical implications in Ewing's sarcoma. Cell Prolif. 2014;48 (1):1-6. doi:10.1111/cpr.12160 
8. Wang S, Tran E. Unexpected functions of IncRNAs in gene regulation. Commun Integr Biol. 2013;6(6):e27610. doi:10.4161/cib.27610

9. Bergmann JH, Spector DL. Long non-coding RNAs: modulators of nuclear structure and function. Curr Opin Cell Biol. 2014;26:10-18. doi:10.1016/j.ceb.2013.08.005

10. Lv L, Xu Y-P, Zhao D, et al. Mitogenic and oncogenic stimulation of K433 acetylation promotes PKM2 protein kinase activity and nuclear localization. Mol Cell. 2013;52(3):340-352. doi:10.1016/j. molcel.2013.09.004

11. Huang M-J, Zhao J-Y, Xu -J-J, Li J, Zhuang Y-F, Zhang X-L. lncRNA ADAMTS9-AS2 controls human mesenchymal stem cell chondrogenic differentiation and functions as a ceRNA. Mol Ther Nucleic Acids. 2019;18:533-545. doi:10.1016/j.omtn.2019.08.027

12. Sanger HL, Klotz G, Riesner D, Gross HJ, Kleinschmidt AK. Viroids are single-stranded covalently closed circular RNA molecules existing as highly base-paired rod-like structures. Proc Natl Acad Sci. 1976;73(11):3852-3856. doi:10.1073/pnas.73.11.3852

13. Jeck WR, Sorrentino JA, Wang K, et al. Circular RNAs are abundant, conserved, and associated with ALU repeats. RNA. 2012;19 (2):141-157. doi:10.1261/rna.035667.112

14. Hansen TB, Jensen TI, Clausen BH, et al. Natural RNA circles function as efficient microRNA sponges. Nature. 2013;495 (7441):384-388. doi:10.1038/nature11993

15. Zhang Z, Wang C, Zhang Y, Yu S, Zhao G, Xu J. CircDUSP16 promotes the tumorigenesis and invasion of gastric cancer by sponging miR-145-5p. Gastric Cancer. 2019.

16. Du WW, Yang W, Li X, et al. A circular RNA circ-DNMT1 enhances breast cancer progression by activating autophagy. Oncogene. 2018;37(44):5829-5842. doi:10.1038/s41388-018-0369-y

17. Zeng Y, Du WW, Wu Y, et al. A circular RNA binds to and activates AKT phosphorylation and nuclear localization reducing apoptosis and enhancing cardiac repair. Theranostics. 2017;7(16):3842-3855. doi:10.7150/thno. 19764

18. Zheng X, Chen L, Zhou Y, et al. A novel protein encoded by a circular RNA circPPP1R12A promotes tumor pathogenesis and metastasis of colon cancer via Hippo-YAP signaling. Mol Cancer. 2019;18(1). doi:10.1186/s12943-019-1010-6.

19. Xia X, Li X, Li F, et al. A novel tumor suppressor protein encoded by circular AKT3 RNA inhibits glioblastoma tumorigenicity by competing with active phosphoinositide-dependent Kinase-1. Mol Cancer. 2019;18(1):131. doi:10.1186/s12943-019-1056-5

20. Yang F, Hu A, Li D, et al. Circ-HuR suppresses HuR expression and gastric cancer progression by inhibiting CNBP transactivation. Mol Cancer. 2019;18(1). doi:10.1186/s12943-019-1094-z.

21. $\mathrm{Hu} \mathrm{X}, \mathrm{Wu} \mathrm{D}, \mathrm{He} \mathrm{X}$, et al. circGSK3 $\beta$ promotes metastasis in esophageal squamous cell carcinoma by augmenting $\beta$-catenin signaling. Mol Cancer. 2019;18(1).

22. Huang X, He M, Huang S, et al. Circular RNA circERBB2 promotes gallbladder cancer progression by regulating PA2G4-dependent rDNA transcription. Mol Cancer. 2019;18(1). doi:10.1186/s12943019-1098-8.

23. Li X, Huang KAI, Yu J. Inhibition of microRNA-21 upregulates the expression of programmed cell death 4 and phosphatase tensin homologue in the A431 squamous cell carcinoma cell line. Oncol Lett. 2014;8(1):203-207. doi:10.3892/ol.2014.2066

24. Darido C, Georgy Smitha R, Wilanowski T, et al. Targeting of the tumor suppressor GRHL3 by a miR-21-dependent proto-oncogenic network results in PTEN loss and tumorigenesis. Cancer Cell. 2011;20(5):635-648. doi:10.1016/j.ccr.2011.10.014

25. Li H, Jiang M, Cui M, et al. MiR-365 enhances the radiosensitivity of non-small cell lung cancer cells through targeting CDC25A. Biochem Biophys Res Commun. 2019;512(2):392-398. doi:10.1016/j. bbrc.2019.03.082

26. Li MIN, Liu L, Zang W, et al. miR-365 overexpression promotes cell proliferation and invasion by targeting ADAMTS-1 in breast cancer. Int J Oncol. 2015;47(1):296-302. doi:10.3892/ijo.2015.3015
27. Yuan F, Liu J, Pang H, et al. MicroRNA-365 suppressed cell proliferation and migration via targeting PAX6 in glioblastoma. Am J Transl Res. 2019;11(1):361.

28. Zhou M, Zhou L, Zheng L, et al. miR-365 promotes Cutaneous Squamous Cell Carcinoma (CSCC) through targeting nuclear factor I/B (NFIB). PLoS One. 2014;9(6):e100620. doi:10.1371/journal. pone. 0100620

29. Zhou M, Liu W, Ma S, et al. A novel onco-miR-365 induces cutaneous squamous cell carcinoma. Carcinogenesis. 2013;34 (7):1653-1659. doi:10.1093/carcin/bgt097

30. Wang C-J, Zhou Z-G, Wang L, et al. Clinicopathological significance of microRNA-31, -143 and -145 expression in colorectal cancer. Dis Markers. 2009;26(1):27-34. doi:10.1155/2009/921907

31. Laurila EM, Kallioniemi A. The diverse role of miR-31 in regulating cancer associated phenotypes. Genes Chromosomes Cancer. 2013;52 (12):1103-1113. doi:10.1002/gcc.22107

32. Wang A, Landén NX, Meisgen F, et al. MicroRNA-31 is overexpressed in cutaneous squamous cell carcinoma and regulates cell motility and colony formation ability of tumor cells. PLoS One. 2014;9(7):e103206. doi:10.1371/journal.pone.0103206

33. Lefort K, Brooks Y, Ostano P, et al. A miR-34a-SIRT6 axis in the squamous cell differentiation network. EMBO J. 2013;32 (16):2248-2263.

34. Xu N, Zhang L, Meisgen F, et al. MicroRNA-125b down-regulates matrix metallopeptidase 13 and inhibits cutaneous squamous cell carcinoma cell proliferation, migration, and invasion. J Biol Chem. 2012;287(35):29899-29908. doi:10.1074/jbc.M112.391243

35. Li W, Wang L, Ji X-B, et al. MiR-199a inhibits tumor growth and attenuates chemoresistance by targeting K-RAS via AKT and ERK signalings. Front Oncol. 2019;9. doi:10.3389/fonc.2019.01071

36. Wang Z, Ma X, Cai Q, et al. MiR-199a-3p promotes gastric cancer progression by targeting ZHX1. FEBS Lett. 2014;588 (23):4504-4512. doi:10.1016/j.febslet.2014.09.047

37. Wang S-H, Zhou J-D, He Q-Y, Yin Z-Q, Cao K, Luo C-Q. MiR-199a inhibits the ability of proliferation and migration by regulating CD44-Ezrin signaling in cutaneous squamous cell carcinoma cells. Int J Clin Exp Pathol. 2014;7(10):7131.

38. Guo X, Xiao H, Guo S, et al. Long noncoding RNA HOTAIR knockdown inhibits autophagy and epithelial-mesenchymal transition through the Wnt signaling pathway in radioresistant human cervical cancer HeLa cells. J Cell Physiol. 2018;234(4):3478-3489. doi: $10.1002 /$ jcp. 26828

39. Han L, Zhang H-C, Li L, Li C-X, Di X, Qu X. Downregulation of long noncoding RNA HOTAIR and EZH2 induces apoptosis and inhibits proliferation, invasion, and migration of human breast cancer cells. Cancer Biother Radiopharm. 2018;33(6):241-251. doi:10.1089/cbr.2017.2432

40. Sand M, Bechara FG, Sand D, et al. Expression profiles of long noncoding RNAs in cutaneous squamous cell carcinoma. Epigenomics. 2016;8(4):501-518. doi:10.2217/epi-2015-0012

41. Yu G-J, Sun Y, Zhang D-W, Zhang P. Long non-coding RNA HOTAIR functions as a competitive endogenous RNA to regulate PRAF2 expression by sponging miR-326 in cutaneous squamous cell carcinoma. Cancer Cell Int. 2019;19(1). doi:10.1186/s12935-0190992-x

42. Kretz M, Siprashvili Z, Chu C, et al. Control of somatic tissue differentiation by the long non-coding RNA TINCR. Nature. 2012;493(7431):231-235. doi:10.1038/nature11661

43. Zhou W, Zhang S, Li J, Li Z, Wang Y, Li X. IncRNA TINCR participates in ALA-PDT-induced apoptosis and autophagy in cutaneous squamous cell carcinoma. $J$ Cell Biochem. 2019;120 (8):13893-13902. doi:10.1002/jcb.28662

44. Chen L, Chen Q, Kuang S, et al. USF1-induced upregulation of LINC01048 promotes cell proliferation and apoptosis in cutaneous squamous cell carcinoma by binding to TAF15 to transcriptionally activate YAP1. Cell Death Dis. 2019;10(4). 
45. Mei X-L, Zhong S. Long noncoding RNA LINC00520 prevents the progression of cutaneous squamous cell carcinoma through the inactivation of the PI3K/Akt signaling pathway by downregulating EGFR. Chin Med J (Engl). 2019;132(4):454-465. doi:10.1097/ CM9.0000000000000070

46. Piipponen M, Nissinen L, Farshchian M, et al. Long noncoding RNA PICSAR promotes growth of cutaneous squamous cell carcinoma by regulating ERK1/2 activity. $J$ Investig Dermatol. 2016;136 (8):1701-1710. doi:10.1016/j.jid.2016.03.028

47. Luo Y, Morgan SL, Wang KC. PICSAR: long noncoding RNA in cutaneous squamous cell carcinoma. J Investig Dermatol. 2016;136 (8):1541-1542. doi:10.1016/j.jid.2016.04.013

48. Ponzio G, Rezzonico R, Bourget I, et al. A new long noncoding RNA (lncRNA) is induced in cutaneous squamous cell carcinoma and down-regulates several anticancer and cell differentiation genes in mouse. J Biol Chem. 2017;292(30):12483-12495. doi:10.1074/jbc. M117.776260

49. Sand M, Bechara FG, Gambichler T, et al. Circular RNA expression in cutaneous squamous cell carcinoma. J Dermatol Sci. 2016;83 (3):210-218. doi:10.1016/j.jdermsci.2016.05.012

50. An X, Liu X, Ma G, Li C. Upregulated circular RNA circ 0070934 facilitates cutaneous squamous cell carcinoma cell growth and invasion by sponging miR-1238 and miR-1247-5p. Biochem Biophys Res Commun. 2019;513(2):380-385. doi:10.1016/j.bbrc.2019.04.017
51. Nelson BR, Makarewich CA, Anderson DM, et al. A peptide encoded by a transcript annotated as long noncoding RNA enhances SERCA activity in muscle. Science. 2016;351(6270):271-275. doi:10.1126/ science.aad4076

52. Anderson Douglas M, Anderson Kelly M, Chang C-L, et al. A micropeptide encoded by a putative long noncoding RNA regulates muscle performance. Cell. 2015;160(4):595-606. doi:10.1016/j. cell.2015.01.009

53. Legnini I, Di Timoteo G, Rossi F, et al. Circ-ZNF609 is a circular RNA that can be translated and functions in myogenesis. Mol Cell. 2017;66(1):22-37.e29. doi:10.1016/j.molcel.2017.02.017

54. Huang J-Z, Chen M, Chen D, et al. A peptide encoded by a putative lncRNA HOXB-AS3 suppresses colon cancer growth. Mol Cell. 2017;68(1):171-184.e176. doi:10.1016/j.molcel.2017.09.015

55. Zhang M, Zhao K, Xu X, et al. A peptide encoded by circular form of LINC-PINT suppresses oncogenic transcriptional elongation in glioblastoma. Nat Commun. 2018;9(1). doi:10.1038/s41467-01806862-2.

56. Liang W-C, Wong C-W, Liang -P-P, et al. Translation of the circular RNA circ $\beta$-catenin promotes liver cancer cell growth through activation of the Wnt pathway. Genome Biol. 2019;20(1). doi:10.1186/ s13059-019-1685-4.

\section{Publish your work in this journal}

OncoTargets and Therapy is an international, peer-reviewed, open access journal focusing on the pathological basis of all cancers, potential targets for therapy and treatment protocols employed to improve the management of cancer patients. The journal also focuses on the impact of management programs and new therapeutic agents and protocols on patient perspectives such as quality of life, adherence and satisfaction. The manuscript management system is completely online and includes a very quick and fair peer-review system, which is all easy to use. Visit http://www.dovepress.com/ testimonials.php to read real quotes from published authors. 